\title{
RELACIONES BIOCLIMATICAS DEL TRIGO EN LOS MONEGROS
}

\author{
Alberto MARTI EZPELETA \\ Universidad de Zaragozá
}

\begin{abstract}
Resumen: Mediante el cáculo de los coeficientes de correlación existentes entre los rendimientos y los valores mensuales de las precipitaciones, las temperaturas máximas y las temperaturas minimas registradas durante el ciclo vegetativo del trigo, se analizan las relaciones bioclimáticas entre este cultivo y el medio climático al que se ve sometido en la comarca de los Monegros. Gracias a este análisis se determinan los períodos críticos durante los cuales el trigo presenta mayor sensibilidad respecto a los factores ambientales.
\end{abstract}

\begin{abstract}
By means the computation of the correlation coeficient between yields and the monthly values of the precipitation, the maximum and minimum temperatures occurred during the wheat vegetative cycle, the bioclimatic environment at the Monegros area are analised. Thanks to that analysis the critical periods when the wheat have more sensibility to the environment factors are determined.
\end{abstract}

Sumario: Introducción.- Materiales y método.- Análisis de los períodos críticos.- Determinación de los umbrales pluviométricos.- Conclusiones.

\section{1.- INTRODUCCION.}

La variación del comportamiento de la planta y de sus exigencias respecto a un factor dado durante el ciclo biológico adquiere su más clara expresión en los períodos de máxima actividad (períodos críticos) y en los de mínima actividad (período de latencia o de reposo). Así, un período crítico, en relación con un factor o fenómeno determinado, queda definido como el intervalo del ciclo vegetativo durante el cual la planta presenta la máxima sensibilidad respecto a este factor. Las variaciones del factor ambiental se reflejan así de modo evidente en el rendimiento, determinando las mayores oscilaciones de éste, tanto en sentido negativo como en sentido positivo, en relación con el factor que se considera (AZZI, 1959).

Del mismo modo podemos definir un período de latencia como el intervalo en el que la planta presenta la mínima sensibilidad en relación con los agentes ambientales. Las variaciones de las temperaturas, de las precipitaciones, etc, no se reflejan apenas en la planta, de manera que las oscilaciones de la producción en relación con la variación de cada uno de los factores climáticos resultan amortiguadas o reducidas. 
El conocimiento de los períodos críticos es de importancia fundamental para orientarse hacia una mejor adaptación de los cultivos al medio climático, a través de dos métodos:

- Regulando la época de la siembra o adoptando una variedad que posea el grado de precocidad suficiente para que el período crítico coincida con un intervalo en el que la frecuencia del fenómeno adverso resulte lo más pequeña posible.

- Concentrando en el período crítico los medios prácticos mediante los cuales se tiende a disminuir la intensidad del fenómeno adverso.

\section{2.- MATERIALES Y METODO.}

Para conocer los períodos en los que los factores ambientales tienen mayor o menor repercusión en la producción del trigo vamos a calcular el grado de dependencia del rendimiento final respecto de los valores mensuales de las precipitaciones, las temperaturas mínimas y las temperaturas máximas durante su ciclo vegetativo (octubre a junio).

Para el cálculo de las relaciones bioclimáticas hemos analizado los coeficientes de correlacion existentes entre los rendimientos y los tres parámetros climáticos mediante correlaciones simples y correlaciones parciales de primer orden. Este método ha sido ya utilizado en algunos estudios agroclimáticos realizados en España (JUAREZ, 1979; LOPEZ, 1988; MARTI, 1991). En Italia se ha utilizado ampliamente desde que fue ideado por Azzi a finales de los años veinte en el Instituto de Ecología Agraria de Perugia (AZZI, 1928); posteriormente ha ido siendo mejorado por sus continuadores dando excelentes resultados con los cultivos de secano de todo el territorio italiano (BALTADORI, 1952; PORCEDU, 1967; PITZALIS, 1974; PANDOLFI e PITZALIS, 1982; PINNOLA et al., 1987).

Debemos tener en cuenta que la obtención de coeficientes de correlación significativos indica sólo que exíste una relación entre los parámetros considerados, lo que permite establecer una interpretación de esta relación de dependencia. Los factores que intervienen en los rendimientos de una planta son diversos, por lo que los resultados obtenidos son un primer intento de hallar una explicación física o biológica de dicha relación (LOPEZ, 1988). Además, esta conexión medio- 
planta es una relación muy compleja y cualquiera que sea el procedimiento empleado para hallarla contendrá siempre un margen de error a tener en cuenta.

Los resultados obtenidos de las correlaciones aparecen reflejados en los cuadros 1 al 4 . La simbología empleada es la siguiente:

RP correlación rendimientos-precipitaciones

Rtm correlación rendimientos-temperaturas mínimas

RTM correlación rendimientos-temperaturas máximas

Cuando se utilizan los coeficientes de correlación parciales de primer órden se enfrentan los rendimientos con otras dos variables y se analiza la dependencia entre las dos primeras eliminando el efecto de la tercera. Así, por ejemplo:

RP.tm: correlación entre los rendimientos y las precipitaciones eliminando el efecto de las temperaturas mínimas.

RTM.P: correlación entre los rendimientos y las temperaturas máximas eliminando el efecto de las precipitaciones.

Las series utilizadas han sido las correspondientes al trigo en Lalueza, Lanaja, Castejón y Leciñena, en un período de años que va desde 1976 a 1988 para Leciñena y desde 1979 a 1988 para las otras tres. A partir de los resultados obtenidos vamos a hacer un análisis de los efectos que los parámetros climáticos utilizados tienen sobre los rendimientos.

\section{3.- ANALISIS DE LOS PERIODOS CRITICOS.}

Los coeficientes obtenidos pueden ser positivos o negativos: en el caso de las correlaciones simples un coeficiente positivo indica un déficit de ese parámetro, y un coeficiente negativo el caso contrario, es decir, un exceso. Cuanto más se acerque el valor obtenido a -1 o a 1 mayor será el exceso o el déficit respectivamente. En el caso de las relaciones parciales de primer órden, un aumento en el coeficiente positivo (el de déficit) indica que la tercera variable beneficia a las otras dos, mientras que si disminuye las perjudica; y en cuanto al coeficiente negativo (el de exceso), si éste disminuye implica que la tercera variable perjudica a las demás, y si aumenta las beneficia. 
a) En Lanaja (Fig. 1) la precipitación muestra un ligero déficit en los meses de diciembre y enero, meses que son decisivos para constituir las reservas utilizadas en fases sucesivas. La correlación entre la suma de las precipitaciones de noviembre y diciembre con los rendimientos da un coeficiente elevado, de 0,75 , lo cual revela la importancia que las lluvias tienen al inicio del ciclo vegetativo del trigo para llevar a cabo su siembra y una buena nascencia. En el mes de febrero se observa un exceso de agua que es perjudicial para el rendimiento, y que permanece constante eliminando el efecto de las temperaturas. En el mes de abril se aprecia otro período con un moderado déficit de precipitación, coincidente con la fase de floración-espigado, que es cuando la planta requiere mayor cantidad de agua. Calculando la correlación entre la precipitación de abril mas la de mayo con el rendimiento el coeficiente es más elevado, dando 0,71 . En estos dos meses junto con noviembre y diciembre se producen en Lanaja dos períodos críticos en cuanto a precipitaciones, durante los cuales la falta de agua va a perjudicar gravemente el rendimiento final del cultivo.

En cuanto a las temperaturas mínimas sólo se aprecian dos períodos en los que el exceso de bajas temperaturas puede perjudicar al cereal, marzo y junio, aunque en una proporción muy baja. También se observa durante los meses de diciembre y enero un pequeño déficit de temperaturas mínimas, período durante el cual se produce el enraizamiento de las plántulas y el posterior ahijamiento, que requieren unas temperaturas mínimas bajas.

El exceso de temperaturas máximas se manifiesta en el mes de junio, que alcanza unos coeficientes moderados, y que sólo descienden al eliminar el efecto de las temperaturas mínimas, lo que implica que éstas son también perjudiciales durante este mes. Estos coeficientes señalan las consecuencias negativas de los valores excesivamente elevados durante este período que pueden producir el asurado o golpe de calor.

b) En Lalueza (Fig. 1) las precipitaciones vuelven a mostrar un importante exceso durante el mes de febrero, con coeficientes altos que se mantienen al eliminar el efecto de los otros dos parámetros. Además muestran un ligero déficit al final del ciclo vegetativo del trigo, en los meses de mayo y junio. En éste último el déficit desaparece al eliminar el efecto de las temperaturas máximas, lo que indica que esa necesidad hídrica viene dada para compensar las elevadas temperaturas que durante este mes se registran, la cual desaparece al suprimir la acción de estas últimas. 
Las temperaturas mínimas vuelven a registrar un déficit moderado durante diciembre y enero. En marzo, por el contrario, se produce la situación inversa, al igual que en mayo, donde el exceso puede causar un perjuicio en los sembrados, que sólo es significativo si se elimina el efecto moderador de las temperaturas máximas.

Las temperaturas máximas no son deficitarias en ningún momento del ciclo vegetativo del trigo, al contrario, su exceso queda constatado en los coeficientes durante varios momentos de dicho ciclo. Al comienzo, en noviembre, diciembre y enero, cuando el cereal necesita de temperaturas bajas para un buen enraizamiento y ahijado, las temperaturas máximas pueden ser perjudiciales, especialmente si se elimina el efecto de las temperaturas mínimas. El otro período en el que tienen un importante efecto negativo, con coeficientes negativos altos, es en mayo y junio, que es cuando pueden dar lugar a la temida ola de calor.

c) En Castejón (Fig. 1) los meses de noviembre y diciembre presentan por separado un ligero déficit de precipitaciones, pero si sumamos los valores de ambos, el coeficiente obtenido de su correlación con los rendimientos es de 0,80. Ello indica la importante influencia que las precipitaciones tienen en este período sobre la cosecha. En febrero vuelve a aparecer el exceso de agua negativo, aunque aquí es menos importante que en Lalueza y en Lanaja. El mes de abril, el mes en que se produce la floración-espigado, registra un déficit moderado que aumenta si le sumamos los valores de mayo, dando un coeficiente de 0,79. Vemos, pues, como aquí, al igual que en Lanaja, son éste junto con noviembre-diciembre los dos períodos del ciclo vegetativo en los que el trigo es más sensible y más exigente en cuanto a precipitaciones.

Las temperaturas mínimas son un poco escasas durante el invierno, lo cual puede perjudicar el buen ahijado del trigo. Más adelante, al reiniciar su crecimiento después del reposo invernal las temperaturas bajas pueden ocasionarle algún peligro por su exceso, aunque no es muy importante.

Las temperaturas máximas no muestran coeficientes significativos, si bien en el mes de junio el exceso de éstas supone cierto perjuicio sobre el rendimiento final. 
d) Por último, en Leciñena (Fig. 1) se producen dos déficits no muy significativos en las precipitaciones de noviembre y mayo, que se corresponden con los períodos de siembra y el de floración, que aquí en Leciñena tiene lugar hacia el 8 de mayo.

En cuanto a las temperaturas mínimas se produce un pequeño déficit en febrero, coincidente con la fase del ahijado que, en esta zona, y debido a la acumulación más lenta de grados-día sufre un retraso con respecto a las otras áreas. En el mes de junio se detecta un exceso, que aumenta al calcular los coeficientes parciales, dando un cifra significativa al restar el efecto de las temperaturas máximas.

Y como viene siendo habitual, las temperaturas máximas registran un moderado exceso al final de la campaña, siendo más elevado al eliminar el efecto de las temperaturas mínimas.

\section{4.- DETERMINACION DE LOS UMBRALES PLUVIOMETRICOS.}

A continuación podemos calcular los umbrales de precipitación en los dos períodos críticos del ciclo vegetativo del trigo (noviembre-diciembre y abril-mayo), por encima y por debajo de los cuales la cosecha resultante será mejor o peor de lo normal respectivamente. Conociendo estos umbrales se podrá evaluar el riesgo de adversidad por falta de precipitación o la probabilidad de obtener una buena cosecha.

Para determinar estos umbrales vamos a tomar los datos pluviométricos y de rendimientos de Castejón y de Lanaja, que como vimos anteriormente mantenían una alta correlación entre sí en esos dos períodos críticos. Hay que tener en cuenta que la serie utilizada tiene 10 años, por lo que los resultados obtenidos sólo son una aproximación a la realidad, la cual es mucho más compleja.

El método utilizado es el de los equivalentes de Azzi (1959), utilizado también por Baltadori (1952). Los datos de las precipitaciones en estos dos períodos y de los rendimientos se llevan a un cuadro de ejes ortogonales, indicando en el eje vertical las lluvias y en el horizontal las campañas agrícolas. En el punto de intersección de las paralelas a los ejes se marcan con un círculo negro los años con cosecha abundante y con un círculo blanco los años con cosecha escasa. Así, mientras que en la parte superior del cuadro tenemos exclusivamente situaciones favorables, en la parte inferior se 
observan sólo situaciones negativas a consecuencia de las escasas precipitaciones durante estos períodos.

Queda una parte intermedia donde los años buenos y los años malos se mezclan, dando como resultado una zona mixta. Esta mezcla de valores se explica muy bien si se considera que la lluvia no actúa por sí sola sino en unión de los demás factores del medio ambiente. El conjunto ambiental puede algunas veces acentuar la evaporación de tal modo que, a pesar de una lluvia abundante, la planta no pueda hacer frente a la demanda de agua que las temperaturas le requieren, manifestándose un estado de sequía. Así ocurre, por ejemplo, cuando a una precipitación abundante sucede un período de vientos secos, algo muy normal en el valle del Ebro. Contrariamente, el conjunto ambiental puede disminuir el efecto de la insuficiencia de precipitaciones de manera que la sequía no llegue a manifestarse a pesar de una relativa escasez de lluvias.

En la Fig. 2 quedan representados los umbrales pluviométricos de Lanaja y Castejón en los períodos críticos del ciclo vegetativo del trigo. Se ha considerado como cosecha buena la que estaba por encima de la media y como cosecha escasa la que estaba por debajo de aquella.

Los umbrales obtenidos son muy similares en las dos zonas. Así, en Lanaja, durante el período de noviembre-diciembre por encima de los $70 \mathrm{~mm}$ todas las cosechas son buenas, mientras que con precipitaciones inferiores a $60 \mathrm{~mm}$ han sido escasas. En Castejón, el valor que separa estos dos grupos es el de $60 \mathrm{~mm}$.

En el período de abril-mayo la zona intermedia es mucho más amplia, lo que implica que en este período otros factores ambientales además de las precipitaciones influyen sobre los rendimientos. En Lanaja con precipitaciones superiores a $122 \mathrm{~mm}$ las cosechas han sido buenas; por debajo de los $55 \mathrm{~mm}$ en este período las cosechas fueron inferiores a la media. Con precipitaciones entre 55 y $122 \mathrm{~mm}$ ha habido años en los que se ha recogido una buena cosecha y otros en los que ha sido mala, debido, como ya hemos dicho, a la acción de otros factores como temperaturas, plagas, vientos fuertes, etc.

En Castejón estos dos umbrales están más cercanos y, así, por encima de los $120 \mathrm{~mm}$ los rendimientos han sido altos, mientras que por debajo de los $70 \mathrm{~mm}$ fueron escasos. 
Es posible trazar una línea que divida en dos grupos los casos favorables y los desfavorables y que, a la vez, separe un número similar de casos asimétricos por encima y por debajo de la misma. El valor de precipitación por donde pase esta línea será la media de los dos umbrales de cada período. Así , en Lanaja este valor es de $65 \mathrm{~mm}$ en noviembre-diciembre y de $88 \mathrm{~mm}$ en abrilmayo. En Castejón estos valores son 60 y $95 \mathrm{~mm}$ respectivamente.

Otro método que puede servir para calcular este umbral es el de la recta de regresión, calculada para los valores de precipitación y de rendimientos en estos períodos:

$$
\begin{array}{lll}
\text { Lanaja } & \begin{array}{l}
\text { noviembre - diciembre : } \\
\text { abril - mayo: } \\
\text { noviembre - diciembre: } \\
\text { abril - mayo: } \mathrm{y}=0,115 \mathrm{x}+2,1
\end{array} & \begin{array}{c}
\mathrm{y}=0,23 \mathrm{x}-1,33 \\
\mathrm{y}=0,13 \mathrm{x}+2,2 \\
\mathrm{y}=0,13 \mathrm{x}+5
\end{array} \\
\text { siendo } & \begin{array}{l}
\mathrm{y}=\text { rendimiento } \\
\mathrm{x}=\text { precipitación de cada período }
\end{array}
\end{array}
$$

Sustituyendo la y por el rendimiento medio de la serie analizada obtenemos para $\mathrm{x}$ los siguientes valores:

$\begin{array}{lll}\text { Lanaja } & \text { noviembre - diciembre: } & 67 \mathrm{~mm} \\ & \text { abril - mayo: } & 87 \mathrm{~mm} \\ \text { Castejón } & \text { noviembre - diciembre: } & 61 \mathrm{~mm} \\ & \text { abril - mayo: } & 94 \mathrm{~mm}\end{array}$

Como vemos los resultados son muy similares a los obtenidos por el método de los equivalentes, por los que podemos considerar estos valores como una estimación de los umbrales pluviométricos en estos dos períodos críticos que separan los años buenos de los años malos, es decir, los años en los que la cosecha es superior o inferior a la media. Calculados estos valores se podrán, posteriormente, evaluar las probabilidades de que las precipitaciones registradas se sitúen por encima o por debajo de estos umbrales, lo que nos revelará el riesgo de una mala cosecha o la probabilidad de que sea buena. 


\section{5.- CONCLUSIONES}

Del análisis previo se constata como durante el ciclo vegetativo del trigo en diversas zonas de secano de los Monegros tienen lugar unos períodos críticos durante los cuales este cultivo presenta mayor sensibilidad a los factores ambientales analizados.

- En cuanto a las precipitaciones, exísten dos períodos muy claros en los que el déficit de agua va a ocasionar una notable disminución del rendimiento final; el primero de estos períodos es el comprendido entre noviembre-diciembre, durante el cual la disponibilidad de agua es imprescindible para una buena nascencia del cereal; el segundo lo encontramos en los meses de abril-mayo, que es cuando tiene lugar la floración-espigado del trigo y cuando presenta las máximas exigencias hídricas. Durante los meses de diciembre y enero también exíste un pequeño déficit debido a la importancia de este período para llenar las reservas hídricas del suelo.

Lo contrario ocurre en el mes de febrero, que presenta en todos los casos un exceso de agua que puede provocar una disminución del rendimiento.

- Respecto a las temperaturas mínimas, éstas repercutirán negativamente en la cosecha final si se presentan en exceso en el mes de marzo y al final del ciclo vegetativo (mayo y junio). Durante los meses del invierno, sin embargo, las bajas temperaturas son beneficiosas para el buen ahijamiento de las plantulas del trigo, siendo perjudicial un déficit de éstas.

- Al final de mayo y principio de junio, cuando el grano está en fase lechosa, el exceso de temperaturas máximas producirá el asurado de los granos con una deshidratación de éstos y una imporatante pérdida de peso.

\section{BIBLIOGRAFIA:}

AZZI, G.(1928): "Influenza dei fattori meteorologici sullo sviluppo e il rendimento della vite in Umbria", Istituto di Ecologia Agraria, Perugia.

AZZI, G.(1959):Ecología agraria, Salvat, Barcelona.

BALTADORI, A.(1952): "Gli equivalenti pluviometrici del Fico", Annali della Facolta di Agraria, Perugia. 
BALTADORI, A (1961), "Il problema della Climatologia Agraria", Atti del Convegno Internazionale Difesa delle Culture e degli Allevamenti dalle avversitá climatiche, Torino, 1961.

DIEHL,R y MATEO BOX, J.M. (1988): Fitotecnia General, Ed. Mundi Prensa, Madrid.

JUAREZ SANCHEZ-RUBIO, C. (1979): Caracteres climáticos de la cuenca del Guadiana y sus repercusiones agrarias, Univ. Salamanca.

LITTLE, T.M. y HILLS, T (1981) :Métodos estadísticos para la investigación en la agricultura, Ed. Trillas, México

LOPEZ BONILLO, D (1988): Los climas de Tarragona y sus repercusiones agrícolas, Publicaciones de la Diputación de Tarragona, Tarragona.

MARTI EZPELETA, A (1991): La Agroclimatología de los Monegros: búsqueda de alternativas al uso tradicional del secano. Memoria de Licenciatura. Instituto de Estudios Altoaragoneses, Col. Microficha, Huesca. (en prensa).

MARTI EZPELETA, A (1991): "Repercusiones de la irregularidad pluviométrica en los rendimientos de los cereales de secano en los Monegros", Rev. ITEA, vol. $88 \mathrm{~V}$ n $^{\circ} 2$ Asociación Interprofesional para el Desarrollo Agrario, Zaragoza. (en prensa).

PANDOLFI, A.M. e PITZALIS, M. (1982): "Fattori estrinseci ed intrinseci e fasi iniziali di sviluppo in varietá di frumento", Rev. Ecologia Agraria, vol. 28 nº 2, Perugia.

PINNOLA, I.M., LORENZETTI, M.C. e BALTADORI, A. (1987): "Bioclimatological relationships of chesnut in the province of Cosenza". Int. Conf. on Agrometeorology, Cesena.

PITZALIS, M. (1974): "Rapporti bioclimatici del frumento ad Ascoli Piceno", Rev. Ecologia Agraria, vol. $10 \mathrm{n}^{\mathrm{o}} 1$, Perugia.

PORCEDU, E. (1967): "Andamento dei fattori climatici e rendimento del frumento nell'ambiente di Perugia", Rev. Ecologia Agraria, vol 3 no 2, Perugia. 
Fig. 1: Evolución de los coeficientes de correlacion simple entre los valores mensuales de los factores ambientales y el rendimiento del trigo para el período 1976-1988.

\section{CASTEJON}

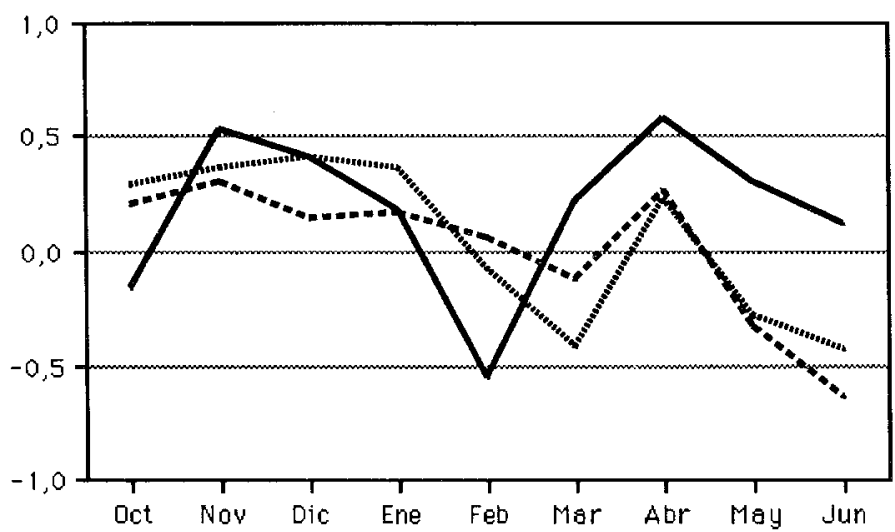

- Precipitaciones

---ne-. T med máxima:

...umunu.... T med mínimas

\section{LALUEZA}
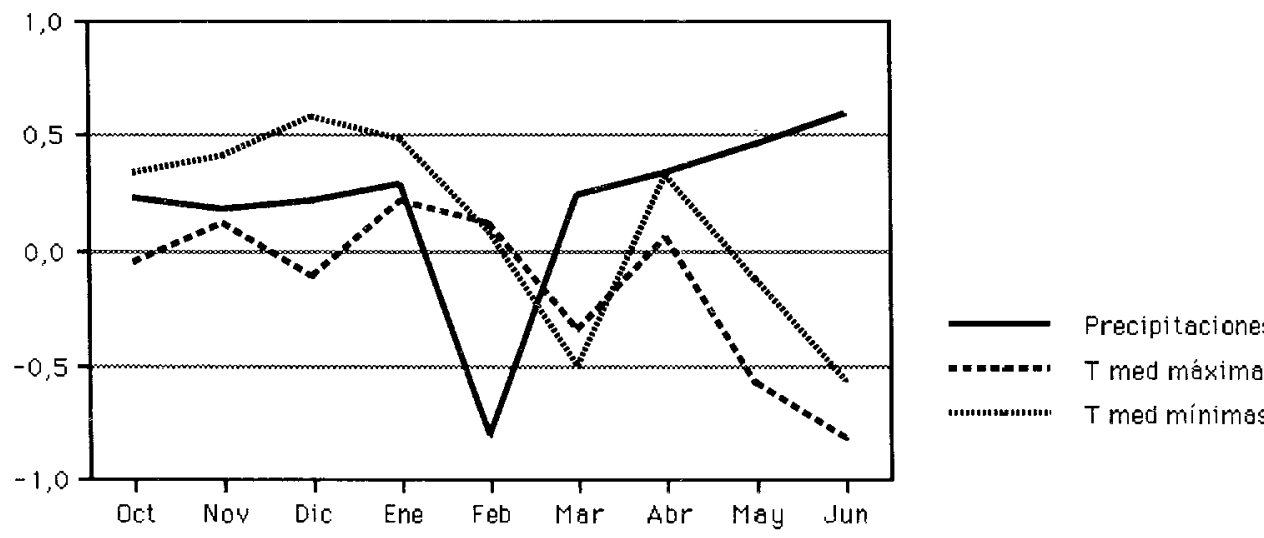

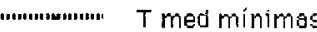




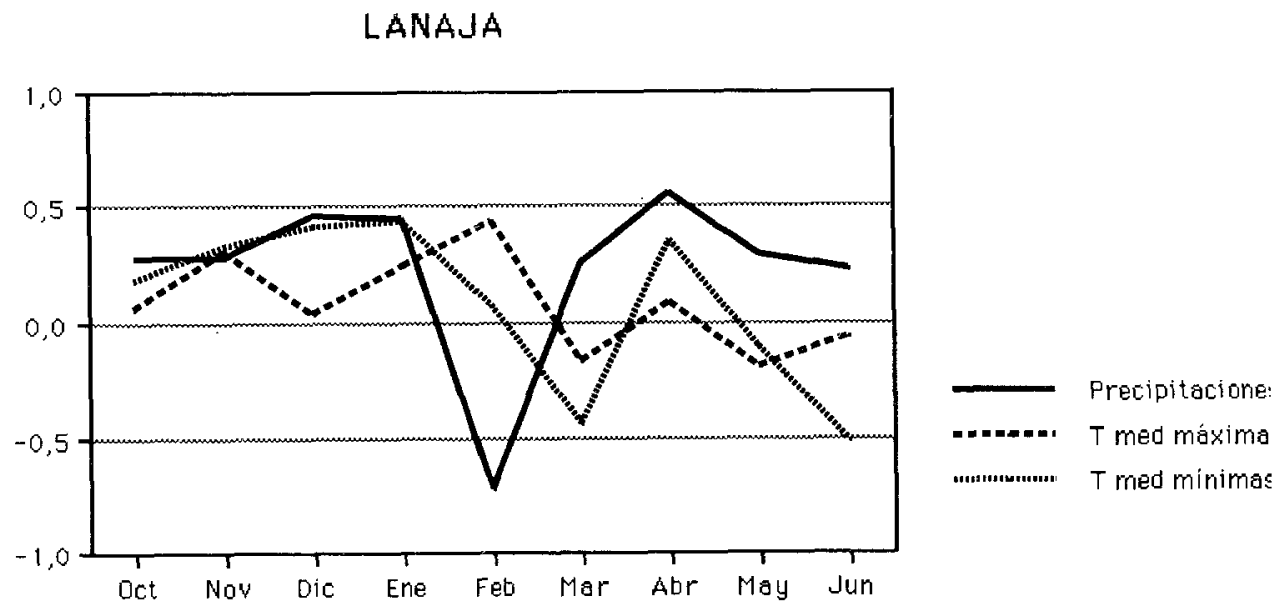

\section{LECIÑNENA}

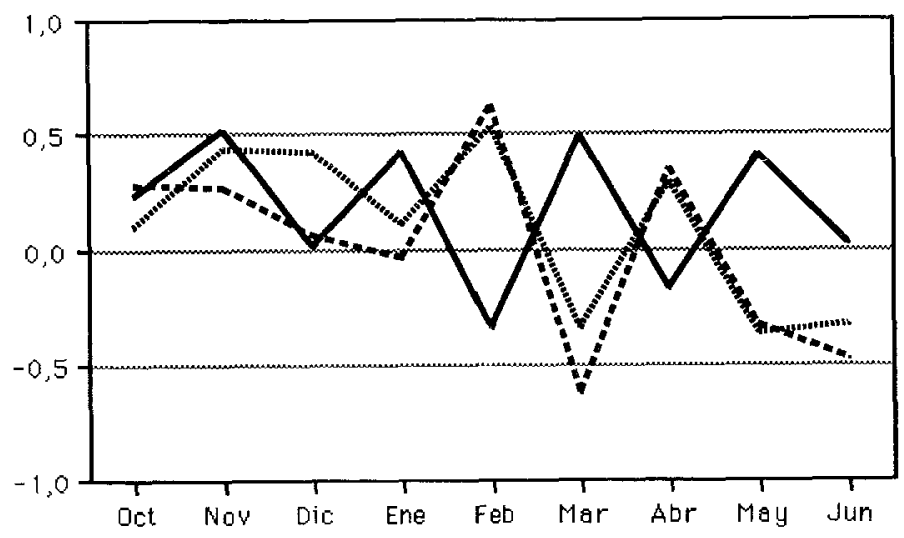

Precipitacione

............... T med minimas 
Fig. 2 : Umbrales pluviométricos en los períodos críticos del trigo.

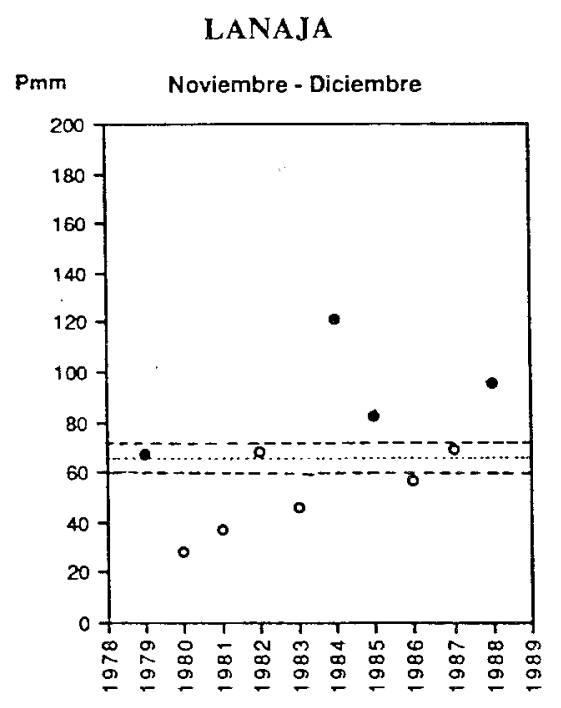

\section{CASTEJON}
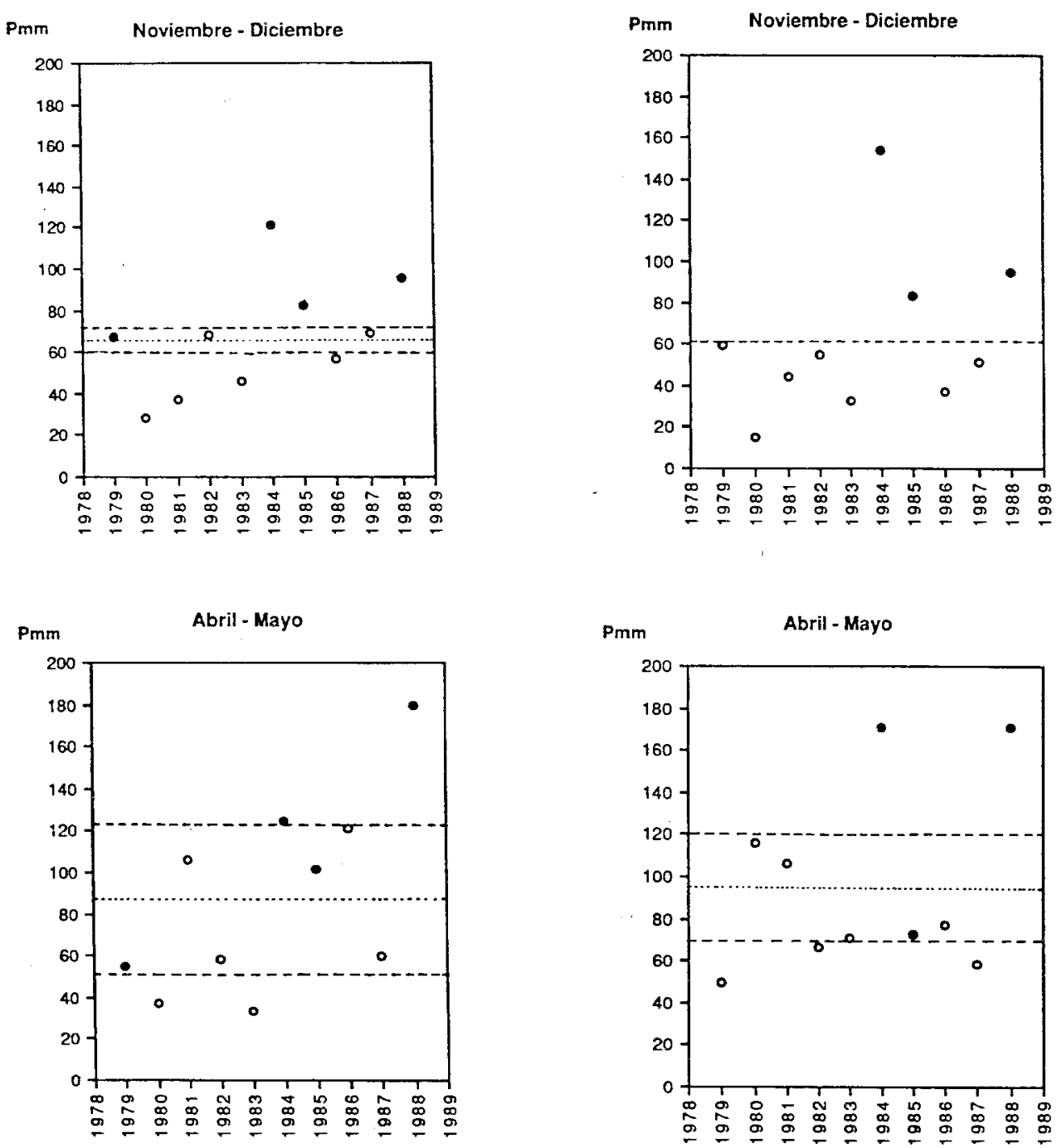


\begin{tabular}{|l||c|c|c|c||c|c|c||c|c|c|}
\hline CUADRO & 1 & \multicolumn{1}{c|}{ ACCION DE LOS ELEMENTOS CLIMATICOS SOBRE EL RENDIMIENTO DEL } \\
\hline TRIGO EN LANAJA \\
\hline MESES & RP & RP.tm & RP.TM & Rtm & Rtm.TM & Rtm.P & RTM & RTM.tm & RTM.P \\
\hline Octubre & 0.27 & 0.21 & 0.36 & 0.18 & 0.17 & 0.06 & 0.06 & 0.02 & 0.2 \\
\hline Noviembre & 0.27 & 0.1 & 0.19 & 0.32 & 0.22 & 0.19 & 0.30 & 0.18 & 0.23 \\
\hline Diciembre & 0.45 & 0.26 & 0.54 & 0.40 & 0.59 & 0.14 & 0.03 & -0.47 & -0.33 \\
\hline Enero & 0.44 & 0.33 & 0.43 & 0.43 & 0.61 & 0.31 & 0.24 & -0.53 & 0.21 \\
\hline Febrero & -0.72 & -0.72 & -0.71 & 0.07 & -0.41 & 0.17 & 0.43 & 0.50 & 0.40 \\
\hline Marzo & 0.25 & -0.06 & 0.18 & -0.44 & -0.44 & -0.38 & -0.18 & 0.20 & 0.06 \\
\hline Abril & 0.55 & 0.52 & 0.63 & 0.34 & 0.38 & 0.27 & 0.08 & -0.21 & 0.37 \\
\hline Mayo & 0.28 & 0.27 & 0.21 & -0.12 & 0.09 & 0.09 & -0.2 & -0.18 & -0.07 \\
\hline Junio & 0.23 & 0.15 & -0.17 & -0.52 & -0.08 & -0.49 & -0.63 & -0.42 & -0.62 \\
\hline
\end{tabular}

CUADRO 32 - ACCION DE LOS ELEMENTOS CLIMATICOS SOBRE EL RENDIMIENTO DEL TRIGO EN LALUEZA

\begin{tabular}{|l||c|c|c||c|c|c||c|c|c|}
\hline \hline MESES & RP & RP.tm & RP.TM & Rtm & Rtm.TM & Rtm.P & RTM & RTM.tm & RTM.P \\
\hline Octubre & 0.23 & 0.12 & 0.29 & 0.33 & 0.33 & 0.27 & -0.06 & -0.07 & 0.2 \\
\hline Noviembre & 0.17 & -0.33 & 0.14 & 0.41 & 0.40 & 0.49 & 0.11 & -0.69 & -0.05 \\
\hline Diciembre & 0.21 & -0.31 & 0.41 & 0.57 & 0.71 & 0.60 & -0.12 & -0.53 & -0.37 \\
\hline Enero & 0.29 & 0.17 & 0.33 & 0.48 & 0.57 & 0.43 & 0.21 & -0.40 & 0.27 \\
\hline Febrero & -0.81 & -0.81 & -0.80 & 0.08 & 0.02 & 0.01 & 0.11 & 0.07 & -0.03 \\
\hline Marzo & 0.24 & 0.01 & -0.03 & -0.50 & -0.39 & -0.45 & -0.35 & 0.08 & 0.26 \\
\hline Abril & 0.33 & 0.41 & 0.51 & 0.32 & 0.40 & 0.40 & 0.05 & -0.25 & 0.41 \\
\hline Mayo & 0.45 & 0.53 & 0.03 & -0.13 & -0.74 & -0.35 & -0.58 & -0.83 & 0.41 \\
\hline Junio & 0.59 & 0.45 & -0.08 & -0.56 & -0.40 & -0.40 & -0.82 & -0.72 & -0.71 \\
\hline
\end{tabular}




\begin{tabular}{|l||c|c|c||c|c|c|c|c|c|c|}
\hline CUADRO & 3 & \multicolumn{1}{c|}{ ACCION DE LOS ELEMENTOS CLIMATICOS SOBRE EL RENDIMIENTO DEL } \\
TRIGO EN CASTEJON & RP & RP.tm & RP.TM & Rtm & Rtm.TM & Rtm.P & RTM & RTM.tm & RTM.P \\
\hline MESES & -0.16 & -0.21 & -0.07 & 0.28 & 0.33 & 0.31 & 0.2 & 0.26 & 0.1 \\
\hline Octubre & 0.53 & 0.42 & 0.48 & 0.36 & 0.26 & -0.05 & 0.3 & 0.16 & 0.15 \\
\hline Noviembre & 0.40 & 0.21 & 0.39 & 0.41 & 0.40 & 0.23 & 0.14 & -0.04 & -0.10 \\
\hline Diciembre & 0.17 & 0.16 & 0.12 & 0.36 & 0.40 & 0.36 & 0.16 & -0.24 & 0.11 \\
\hline Enero & -0.55 & -0.56 & -0.55 & -0.08 & -0.10 & -0.15 & 0.06 & 0.06 & 0.04 \\
\hline Febrero & 0.21 & -0.12 & 0.17 & -0.42 & -0.42 & -0.39 & -0.13 & 0.12 & -0.05 \\
\hline Marzo & 0.58 & 0.61 & 0.69 & 0.23 & 0.11 & 0.3 & 0.26 & 0.17 & 0.51 \\
\hline Abril & 0.30 & 0.19 & 0.18 & -0.28 & 0.08 & -0.15 & -0.33 & -0.20 & -0.22 \\
\hline Mayo & 0.11 & -0.04 & 0.01 & -0.43 & -0.25 & -0.42 & -0.64 & -0.57 & -0.64 \\
\hline Junio & &
\end{tabular}

CUADRO 4 - ACCION DE LOS ELEMENTOS CLIMATICOS SOBRE EL RENDIMIENTO DEL TRIGO EN LECIÑENA

\begin{tabular}{|l||c|c|c||c|c|c||c|c|c|}
\hline \hline MESES & RP & RP.tm & RP.TM & Rtm & Rtm.TM & Rtm.P & RTM & RTM.tm & RTM.P \\
\hline Octubre & 0.23 & 0.22 & 0.37 & 0.09 & -0.04 & 0.06 & 0.27 & 0.26 & 0.3 \\
\hline Noviembre & 0.52 & 0.45 & 0.55 & 0.43 & 0.41 & -0.07 & 0.26 & -0.22 & -0.02 \\
\hline Diciembre & 0.01 & 0.32 & 0.01 & 0.42 & 0.45 & 0.51 & 0.05 & -0.19 & 0.05 \\
\hline Enero & 0.42 & 0.41 & 0.42 & 0.10 & 0.24 & 0.03 & -0.04 & -0.22 & -0.07 \\
\hline Febrero & -0.34 & -0.41 & -0.39 & 0.53 & -0.11 & 0.57 & 0.63 & 0.41 & 0.65 \\
\hline Marzo & 0.49 & 0.40 & 0.17 & -0.34 & 0.18 & -0.40 & -0.63 & -0.58 & -0.48 \\
\hline Abril & -0.18 & -0.16 & -0.04 & 0.28 & 0.06 & 0.27 & 0.35 & 0.22 & 0.3 \\
\hline Mayo & 0.40 & 0.36 & 0.46 & -0.37 & -0.56 & -0.32 & -0.33 & -0.46 & -0.27 \\
\hline Junio & 0.02 & -0.39 & 0.20 & -0.33 & -0.78 & -0.49 & -0.48 & -0.76 & -0.29 \\
\hline
\end{tabular}

